\title{
Dynamic avalanche breakdown of a $p-n$ junction: deterministic triggering of a plane streamer front
}

\author{
Pavel Rodin* and Igor Grekhov \\ Ioffe Physicotechnical Institute, Politechnicheskaya 26, 191041, St.-Petersburg, Russia
}

(Dated: September 1, 2018)

\begin{abstract}
We discuss the dynamic impact ionization breakdown of high voltage $p$ - $n$ junction which occurs when the electric field is increased above the threshold of avalanche impact ionization on a time scale smaller than the inverse thermogeneration rate. The avalanche-to-streamer transition characterized by generation of dense electron-hole plasma capable to screen the applied external electric field occurs in such regimes. We argue that the experimentally observed deterministic triggering of the plane streamer front at the electric field strength above the threshold of avalanche impact ionization but yet below the threshold of band-to-band tunneling is generally caused by field-enhanced ionization of deep-level centers. We suggest that the process-induced sulfur centers and native defects such as EL2, HB2, HB5 centers initiate the front in Si and GaAs structures, respectively. In deep-level free structures the plane streamer front is triggered by Zener band-to-band tunneling.
\end{abstract}

PACS numbers: 85.30.-z,72.20.Ht, 71.55.-i

Impact ionization generally leads to avalanche breakdown of semiconductor $p-n$ junctions at high reverse biases. For common breakdown mode the space charge of avalanche carriers drastically modifies the electric field profile, but the voltage $U$ at the $p-n$ junction remains close to the stationary breakdown voltage $U_{b} \stackrel{1}{\underline{1}}$ Different breakdown mode occurs when the electric field is rapidly increased on a time scale smaller than the inverse rate of thermogeneration ${ }^{2.3}$ In absence of thermal carriers in depleted $p-n$ junction the voltage at the structure $U$ continues to increase far above $U_{b}$ before impact ionization sets in ${ }^{2.3}$ Then $U$ rapidly decreases to a negligible residual value $U_{\text {res }} \ll U_{\mathrm{b}}$ because the impact ionization front passes across the structure and fills it with dense electron-hole plasma. Initiation of such front manifests the avalanche-to-streamer transition which is known in gas discharge $e^{\frac{4}{\underline{*}}}$ and semiconductor physics ${ }^{\underline{\underline{5}}}$ mostly with respect to finger-like streamers. Streamer breakdown is characterized by full screening of the applied electric field by the avalanche carriers and occurs when the Maxwell relaxation time in the avalanche becomes smaller than the inverse impact ionization rate.$^{\frac{5}{n}}$ In high voltage $p^{+}$$n-n$ structures the plane TRAPATT-like ionization front moves from the $p^{+}-n$ junction into depleted $n$ base faster than the saturated carrier velocity $v_{s}^{2.3}$ and is generally regarded as a plane streamer front $\underline{\underline{6}}$ This process has found important applications in pulse power electronics?

In Si and GaAs structures the onset of plane streamer front occurs at the electric field strength $F_{\text {th }}$ above the threshold of avalanche impact ionization $F_{\mathrm{a}}$ but below the threshold of Zener band-to-band tunneling $F_{Z}$ (e.g., in Si $F_{\mathrm{a}} \sim 2 \cdot 10^{5} \mathrm{~V} / \mathrm{cm}$ and $F_{\mathrm{Z}} \sim 10^{6} \mathrm{~V} / \mathrm{cm}$, whereas

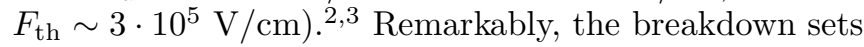
in a pronounced deterministic way: triggering voltage and time at identical driving circuit conditions are not only reproducible, but their variation (jitter) is even below the measurement accuracy ${ }^{2.3}$ This implies the existence of a certain deterministic field-dependent mechanism which supplies the initial carriers to the depleted part of the structure. This mechanism is not yet identified (e.g., see Ref. 8 and discussion therein). In this Letter we argue that plane streamer fronts are initiated by the field enhanced ionization of deep-level midgap electron traps in the depleted part of the $p-n$ junction. We suggest that in Si structure this occurs due to the processinduced deep-level sulfur center, whereas in GaAs structures the native defects such as EL2, HB2 and HB5 centers are responsible for that. In defect-free structures the plane streamer front is initiated by band-to-band tunneling in accordance to the recent theoretical prediction and experimental observation of tunneling-assisted impact ionization fronts $\frac{9,10}{}$

Let us consider a typical high voltage $p^{+}-n-n^{+}$structure with low-doped $n$ base $\left(N_{d} \sim 10^{14} \mathrm{~cm}^{-3}\right.$ for Si and $10^{16} \mathrm{~cm}^{-3}$ for GaAs) of a characteristic width $100 \mu \mathrm{m}$ At the very beginning the structure is in equilibrium. The midgap electron levels in the $n$ base are expected to be occupied. Then the initial reverse bias $U_{0}$ is quasistatically applied to the structure connected in series with load resistance $R$. During the waiting period $-t_{d}<t<0$ [Fig. 1(a)] some deep centers may emit electrons, but the emission rate $e$ at low electric fields is too small to change the occupation of midgap centers significantly. At $t=0$ the steep voltage $\operatorname{ramp} V(t)=U_{0}+A \cdot t$ is applied, where $A$ is typically of the order of $1 \ldots 10 \mathrm{kV} / \mathrm{ns}^{2.3 .9 .10}$ The electric field $F$ in the $n$ base increases and overcomes the effective threshold of avalanche impact ionization $F_{\mathrm{a}}$ on a nanosecond scale [Fig. 1(b)]. However due to the absence of initial carriers the impact ionization does not occur immediately and the electric field keeps increasing. The ionization probability of deep levels $e(F)$ is stimulated by electric field due to the phonon-assisted tunneling mechanism that proceeds to direct tunneling mechanism in higher electric fields ${ }^{11}$ Since $e(F)$ dependence is very steep, the emission of free carriers abruptly starts at a certain electrical field $F>F_{\text {a }}$ triggering propagation of a plane streamer front at $t=t_{0}$. To ensure deterministic triggering, the initial carriers should be distributed uni- 

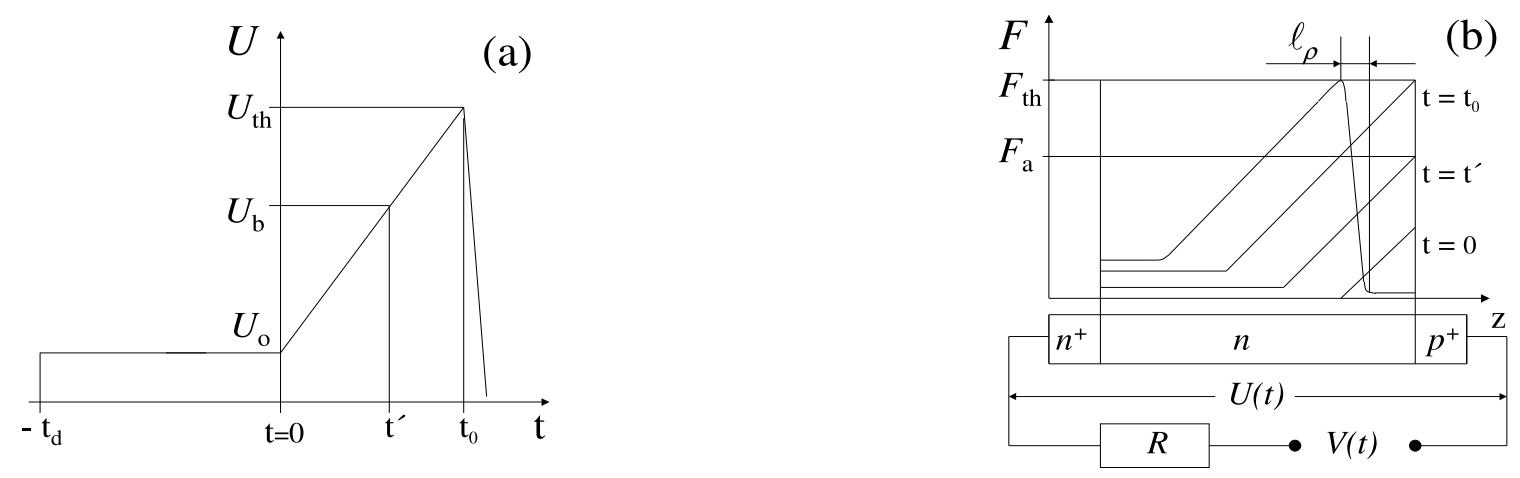

FIG. 1: Sketches of the voltage at the $p^{+}-n-n^{+}$structure $U(t)$ (a) and distributions of the electric field $F(z, t)$ in the $n-$ base (b). For simplicity we assume that $V(t) \equiv U(t)$ during the period $0<t<t_{0}$, neglecting the displacement current. The electric field profile is shown at $t=0$, at $t=t^{\prime}$ when the voltage of stationary breakdown $U_{b}$ is reached and at $t=t_{0}$ when the front is triggered. $F_{\mathrm{a}}$ and $F_{\mathrm{th}}$ are the effective threshold of avalanche impact ionization and the maximum electric field achieved at the moment when the front is triggered. The thin line in panel (b) shows the field profile in the traveling front for $t>t_{0}$. The width of the charged layer at the front tip that screens the electric field is denoted as $\ell_{\rho}$.

formly over the device area on a scale determined by an internal characteristic dimension of the traveling front. This scale can be defined as a width $\ell_{\rho} \sim 10 \mu \mathrm{m}$ (Ref. 8) of the charged layer between the high-field nonconductive region and the low-field conductive region [Fig. 1(b)]. Hence $n_{0} \sim \ell_{\rho}^{-3} \sim 10^{9} \mathrm{~cm}^{-3}$ estimates the threshold concentration of initial carriers.

Two decades after the effect had been discovered, it was realized that the common Soviet fabrication technology 12 used to manufacture high voltage Si structures exhibiting superfast switching ${ }^{2}$ creates a certain deep-level defect in the low-doped $n$ base ${ }^{13}$ This processinduced (PI) defect, previously known in Si and regarded as a recombination center, $\stackrel{14}{,}$ later on was proved to be an electron trap with negligible recombination activity, thus having no effect on the carriers lifetime, and identified as sulfur (S) impurity center $\stackrel{13}{\underline{T}}$ This double donor (see inset in Fig. 2) with ionization energies $\left(E_{c}-0.28\right) \mathrm{eV}$ for the upper midgap $S^{0}$ state (U level) and $\left(E_{c}-0.54\right) \mathrm{eV}$ for the midgap $S^{+}$state (M level) appears in concentrations $N_{P I}=10^{11} \ldots 10^{13} \mathrm{~cm}^{-3} \underline{13}$ Taking into consideration the field-enhanced ionization of PI centers, we are able to resolve the long-standing puzzle of deterministic triggering of plane streamer fronts in Si structures. The emission rate $e(F)$ of this PI center has been experimentally measured only for $F$ up to $10^{5} \mathrm{~V} / \mathrm{cm}, \frac{14}{\underline{1}}$ so we use the results of the semiclassical theory 11 to evaluate $e(F)$ for the relevant field strength $F \sim 2 \ldots 4 \cdot 10^{5} \mathrm{~V} / \mathrm{cm}$. At room temperature only $\mathrm{M}$ levels $(0.54 \mathrm{eV})$ are occupied. The curve 1 in Fig. 2 presents the emission rate $e(F)$ for the $\mathrm{M}$ level at $T=300 \mathrm{~K}$ calculated according to the analytical expression 11

$$
\begin{gathered}
e(F)=e(0) \exp \left(\frac{F^{2}}{F_{c}^{2}}\right) \exp \left(\frac{2 \sqrt{2 m E_{\mathrm{B}}}}{q \tau_{2} F} \ln \frac{12 F^{2}}{F_{c}^{2}}\right), \\
F_{c}^{2} \equiv 3 m \hbar /\left(q^{2} \tau_{2}^{3}\right), \quad 2 \tau_{2} / \hbar \equiv 1 / k T+2 \tau_{1} / \hbar
\end{gathered}
$$

which describes phonon-assisted tunneling with the positive charge of the ionized double donor taken into account. Here $e(0) \approx 2 \cdot 10^{2} \mathrm{~s}^{-1}$ is the emission rate in zero electric field, $\stackrel{13.14}{=}$ and $q$ are the effective mass and the charge of the electron, $k$ is Boltzmann constant, $T$ is the lattice temperature, $E_{\mathrm{B}}$ is the Bohr energy, $\tau_{1}$ is the tunneling time of the vibrational subsystem determined by the energy $\varepsilon_{\mathrm{ph}}$ of the local phonon mode $\varepsilon_{\mathrm{ph}} / k \sim \hbar / 2 k \tau_{1} \sim 1000 \mathrm{~K} \stackrel{11}{11}$ We find that $e(F) \sim 10^{6} \mathrm{~s}^{-1}$ for $F \sim 3 \cdot 10^{5} \mathrm{~V} / \mathrm{cm}$ (Fig. 2). For $N_{P I}=10^{12} \mathrm{~cm}^{-3}$ the total emission rate is $G=N_{P I} e(F) \sim 10^{18} \mathrm{~s}^{-1} \mathrm{~cm}^{-3}$, and the initial concentration $n_{0} \approx 10^{-9} \mathrm{~cm}^{-3}$ can be reached within $\Delta t \approx n_{0} / N_{P I} e(F) \sim 1 \mathrm{~ns}$. This is about the time it takes for the voltage $U$ to increase from the stationary breakdown voltage $U_{\mathrm{b}} \approx 2 \mathrm{kV}$ to the triggering voltage $U_{\mathrm{th}} \approx 3 \mathrm{kV}$ for the typical voltage $\operatorname{ramp} A=1 \mathrm{kV} / \mathrm{ns}^{2}$ Hence for the room temperature the front can be deterministically triggered due to the phonon-assisted tunneling of electrons bound on the M level of the PI center. The emission rate $e(F)$ increases with $T$, and for $T \gtrsim 350 \mathrm{~K}$ bound electrons are prematurely emitted in electrical fields below $F_{a}$. This prevents triggering the streamer front and leads to common avalanche breakdown.

For low temperatures $T \lesssim 200 \mathrm{~K}$ all PI centers are in the ground state: the $\mathrm{U}$ level is occupied and the $\mathrm{M}$ level is empty. Curve 2 in Fig. 2 shows the emission rate for the U level at low temperature when ionization occurs via direct tunneling. The rate is evaluated according to 11

$$
\begin{aligned}
e(F) & =\frac{q F}{\sqrt{8 m E_{0}}} \exp \left(-\frac{F_{0}}{F}\right) \exp \left(2 \sqrt{\frac{E_{B}}{E_{0}}} \ln \frac{6 F_{0}}{F}\right), \\
F_{0} & \equiv 4 \sqrt{2 m E_{0}^{3}} / 3 q \hbar .
\end{aligned}
$$

Here $E_{0}=0.28 \mathrm{eV}$ is the energy of the respective deep level. Similar to the room temperature case, we find 


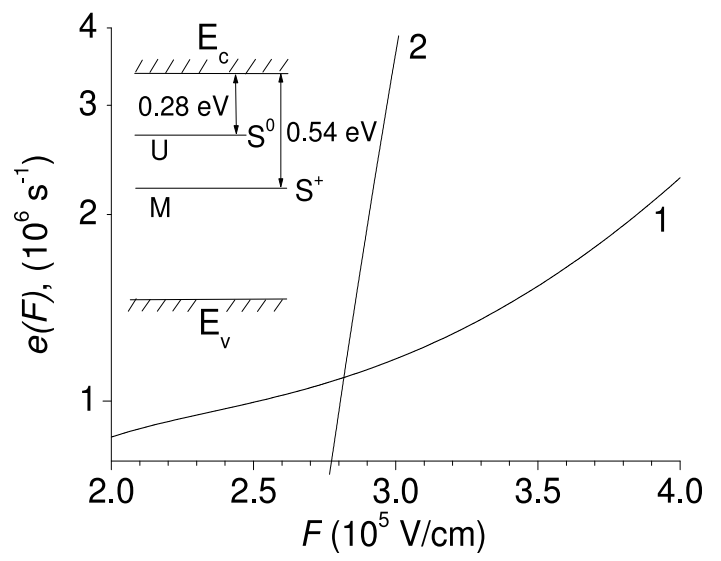

FIG. 2: The ionization rate $e$ of the midgap M level $(0.54 \mathrm{eV})$ at $\mathrm{T}=300 \mathrm{~K}$ (curve 1$)$ and upper midgap $\mathrm{U}$ level $(0.28 \mathrm{eV})$ at $\mathrm{T}=77 \mathrm{~K}$ (curve 2 ) of the process-induced deep-level sulfur center in $\mathrm{Si}$ as a function of the electric field strength $F$. The inset illustrates the position of respective levels in the gap and the charge states of the double donor S impurity.

$e(F) \sim 10^{6} \mathrm{~s}^{-1}$ at $F \sim 3 \cdot 10^{5} \mathrm{~V} / \mathrm{cm}$, although the $e(F)$ dependence is much more steep. We conclude that at low temperatures the front can be deterministically triggered due to direct tunneling of electrons bound on the $\mathrm{U}$ level. This triggering mechanism remains unchanged up to zero temperature. In experiments deterministic triggering of superfast fronts has been shown to disappear for $T>370 \mathrm{~K}$ and proven to sustain at low temperatures up to $77 \mathrm{~K}, 15$ in agreement with our analysis. Details of the calculations will be reported elsewhere. Our findings suggest that concentration of deep-level sulfur centers should be carefully controlled in fabrication of $\mathrm{Si}$ power devices used in pulse sharpening applications $\underline{\underline{7}}$ to ensure efficient and reliable operation.

Unlike high purity $\mathrm{Si}$, where the existence of deep- levels that are capable to trigger the superfast front should be regarded as a rare lucky exception, semi insulating GaAs is a compensated material which possesses deep levels in large concentrations $\frac{16}{16}$ The most known defect is the EL2 deep-level donor $\left(E_{c}-0.75 \mathrm{eV}\right)$ which is present in concentration $\sim 10^{16} \mathrm{~cm}^{-3}$ for free carrier concentration up to $10^{15} \mathrm{~cm}^{-3}$ in $n$ type GaAs $\frac{16}{\underline{16}}$ In $p^{+}-p-n-n^{+}$structures studied in Ref. 3 deep-level acceptors HL5 $\left(E_{v}+0.41 \mathrm{eV}\right)$ and HB2 $\left(E_{v}+0.68 \mathrm{eV}\right)^{16}$ are present in concentrations $10^{14} \ldots 10^{15} \mathrm{~cm}^{-3}, 18$ Below we consider the EL2 defect to illustrate possible triggering mechanism in GaAs-based $p^{+}-n-n^{+}$structures. Due to the exact midgap position the EL2 level in $n$ type GaAs is occupied in equilibrium. It remains occupied after any realistic waiting period $t_{d}$ (typically $t_{d} \sim 100 \mu \mathrm{s}$ ) since its lifetime at zero-electric field is about $10 \mathrm{~s}: \frac{17}{\mathrm{n}}$ The ionization rate $e(F)$ for the EL2 defect is known for electrical fields up to $4 \cdot 10^{5} \mathrm{~V} / \mathrm{cm} .11 .17$ According to Ref. $17 e(F) \sim 10^{3} \mathrm{~s}^{-1}$ at $F=4 \cdot 10^{5} \mathrm{~V} / \mathrm{cm}$. For $N_{E L 2}=10^{16} \mathrm{~cm}^{-3}$ the threshold initial concentration $n_{0}$ can be reached within $\Delta t \approx n_{0} / N_{E L 2} e(F) \sim 100 \mathrm{ps}$, in a reasonable agreement with experiments $\underline{\underline{3}}$

In deep-level-free structures the threshold of the Zener band-to-band tunneling $F_{Z}$ can be reached, provided that the voltage $\operatorname{ramp} A$ is sufficiently large. In this case the plane streamer front is initiated by band-to-band tunneling and propagates due to the combined action of band-to-band tunneling and impact ionization. Such tunneling-assisted impact ionization fronts have been described theoretically ${ }^{9}$ and observed experimentally ${ }^{10}$.

Acknowledgements.- We are indebted to E. Astrova, I. Merkulov, V. Perel', A. Rodina and A. Rozkov for enlightening discussions. This work was supported by the Russian Program for the Support of Research Schools. P. Rodin is grateful to A. Alekseev for hospitality at the University of Geneva and acknowledges the support of the Swiss National Science Foundation.
* Electronic address: rodin@mail.ioffe.ru

1 S.M. Sze, Physics of Semiconductor Devices (Wiley, New York, 1991).

${ }^{2}$ I.V. Grekhov and A.F. Kardo-Sysoev, Sov. Tech. Phys. Lett. 5, 395 (1979).

3 Zh.I. Alferov, I.V. Grekhov, V.M. Efanov, A.F. Kardo-Sysoev, V.I. Korol'kov, and M.N. Stepanova Sov. Tech. Phys. Lett. 13, 454 (1987).

4 E.M. Bazelyan and Yu.P. Raizer, Spark Discharges (CRS, New York, 1998)

5 M.I. D'yakonov and V.Yu. Kachorovskii, Sov. Phys. JETP 67, 1049 (1988).

6 U. Ebert, V. van Saarloos, and C. Caroli, Phys. Rev. E 49, 1530 (1997).

7 I.V. Grekhov, Solid-State Electron. 32, 923 (1989); R.J. Focia, E. Schamiloghu, C.B. Fledermann, F.J. Agee and J. Gaudet, IEEE Trans. Plasma Sci. 25, 138 (1997).

8 P. Rodin, U. Ebert, W. Hundsdorfer and I. Grekhov,
J. Appl. Phys. 92, 1971 (2002).

9 P. Rodin, U. Ebert, W. Hundsdorfer and I. Grekhov, J. Appl. Phys. 92, 958 (2002).

10 S.K. Lyubutin, S.N. Rukin, B.G. Slovikovsky, S.N. Tsyranov, Tech. Phys. Lett. 31, 196 (2005).

11 V.N. Abakumov, V.I. Perel', and I.N. Yassievich, Nonradiative recombination in semiconductors, edited by V. M. Agranovich and A. A. Maradudin, Modern Problems in Condenced Matter Sciences Vol.33, (North-Holland, Amsterdam, 1991).

12 I.V. Grekhov et al, The method to create a source of $\mathrm{Al}$ diffusion in Si, USSR patent N 176989, priority from July 6th 1964.

13 E.V. Astrova, V.B. Voronkov, V.A. Kozlov and A.A. Lebedev, Semicond. Sci. Technol. 13, 488-495 (1998).

14 L.D. Yau and C.T. Sah, Solid-State Electronics 17, 193 (1974); J. Appl. Phys. 46, 1767 (1975).

15 I.V. Grekhov, A.F. Kardo-Sysoev, L.S. Kostina, and S.V. 
Shenderey, Sov. Phys. Tech. Phys. 26, 984 (1981).

16 J.C. Bourgoin, H.J. von Bardeleben, and D. Stie'venard, J. Appl. Phys. 64, R65 (1988).

17 S. Makram-Ebeid, M. Lannoo, Phys. Rev. B 25, 6406 (1982).

18 V.I. Korol'kov, A.V. Rozkov, F.Yu. Soldatenkov and
K.V. Yevstigneyev, Investigation of temperature switching stability of AlGaAs/GaAs-based high voltage superfast switches, Proceedings of the 4th International Seminar on Power Semiconductors ISPS'98, Prague, 2-4 September 1998, pp.163-168. 